\title{
FH - HES
}

\section{Recyclage des oxydes de chrome hexavalent contenus dans les boues galvaniques}

\author{
Patrick Raetzo*
}

\begin{abstract}
Hexavalent Chromium Oxides Recycling from Industrial Sludges
Abstract: In view of a sustainable development, the recycling of chromium (VI) oxides from industrial sludges shows very interesting potential. The proposed process is based on solvent extraction followed by crystallization, the first results show good selectivities and the promise of economical efficency.
\end{abstract}

Keywords: Chromium · Extraction $\cdot$ Recycling $\cdot$ Sludge $\cdot$ Solvent

Le chromage des pièces métalliques a des avantages notables, hors de la spécificité de ce dépôt extrêmement dur pour les pièces en roulement. En effet, l'économie réalisée par la stabilité vis-à-vis de la corrosion, ainsi que la sécurité engendrée des fonctions de ces pièces ainsi chromées, font de ce procédé galvanoplastique un outil remarquable sinon indispensable à toute l'activité technologique moderne. Cependant, ce procédé nécessite l'utilisation d'oxyde de chrome (VI), espèce chimique toxique et extrêmement soluble dans l'eau. Les résidus de cette exploitation contiennent du chrome hexavalent, soit en solution aqueuse à des titres divers, soit sous forme de boues déshydratées ou de précipités hydratés. La Fig. 1 indique un exemple de composition d'une telle boue provenant d'un atelier de chromage. Le recyclage des effluents chromiques est aujourd'hui principalement effectué au moyen de ré-

\footnotetext{
${ }^{*}$ Correspondence: P. Raetzo

Laboratoire de Génie chimique

Ecole d'Ingénieurs de Genève

4 , rue de la Prairie

$\mathrm{CH}-1202$ Genève

Tel.: +4122 3380568

Fax: +4122 3380410

E-Mail: raetzo@eig.unige.ch
}

sines échangeuses d'ions, ou, et bien que moins largement appliquée en particulier pour les concentrés, par voie électrochimique. A titre de comparaison, l'épuration par échange d'ions de 1.2 tonnes d'effluents contenant $3 \mathrm{kM}$ de $\mathrm{CrO}_{3}$ et $1 / 6 \mathrm{kM}$ de fer trivalent, coûte environ $2.34 \mathrm{GJ}$ et produit entre 72 et $180 \mathrm{~kg}$ de boues chromiques ultimes. La voie électrochimique n'est pas moins gourmande en énergie, mais permet de régénérer également le chrome (III) sous forme de chrome (VI) et de produire moitié moins de boues ultimes débarrassées de tout chrome hexavalent. Les boues chromiques résiduelles peuvent être soit réduites chimiquement, soit enfouies en dépôts agréés pour l'entreposage des déchets spéciaux. Le traitement physico-chimique consomme de larges quantités de réactifs et augmente notablement le volume de boues décontaminées ainsi propres à une stabilisation. Du point de vue d'un développement durable, il serait judicieux de comptabiliser l'énergie consommée pour l'extraction minière, d'effectuer un bilan du $\mathrm{CO}_{2}$ produit lors de l'affinage et de mesurer les impacts environnementaux de la consommation des ressources et des résidus produits lors de cette exploitation. Nous avons évalué à respectivement $28.8 \mathrm{GJ}$ et $170 \mathrm{kMoles}$ de $\mathrm{CO}_{2}$ par tonne de $\mathrm{CrO}_{3}$, sans compter les opérations d'extraction mécanique et de broyage.

L'objet de la présente étude, projet cofinancé par la réserve stratégique des HES-SO, s'attelle à l'évaluation d'un procédé d'extraction liquide-solide au moyen de solvants organiques pour permettre le recyclage du $\mathrm{CrO}_{3}$ contenu dans ces déchets solides, et d'en permettre ainsi la réutilisation comme matière première pour l'industrie de la galvanoplastie.

\section{Schéma de recyclage}

Les premiers essais réalisés dans la phase exploratoire montrent une grande sélectivité d'extraction des boues chromiques, sous forme de $\mathrm{CrO}_{3} . \mathrm{S}$ selon le solvant S utilisé. Seuls le fer (III) et l'acide sulfurique demeurent difficiles à séparer du produit recyclé, voir Fig. 2. Ainsi la quantité d'eau résiduelle contenue dans le milieu réactionnel paraît être un paramètre critique lors de la purification, en terme de sélectivité.

Nous proposons le schéma suivant pour les étapes de recyclage des solides contenant au moins $5 \%$ masse de chrome total, le cas le plus défavorable présentera une teneur en chrome (VI) proche de zéro. 


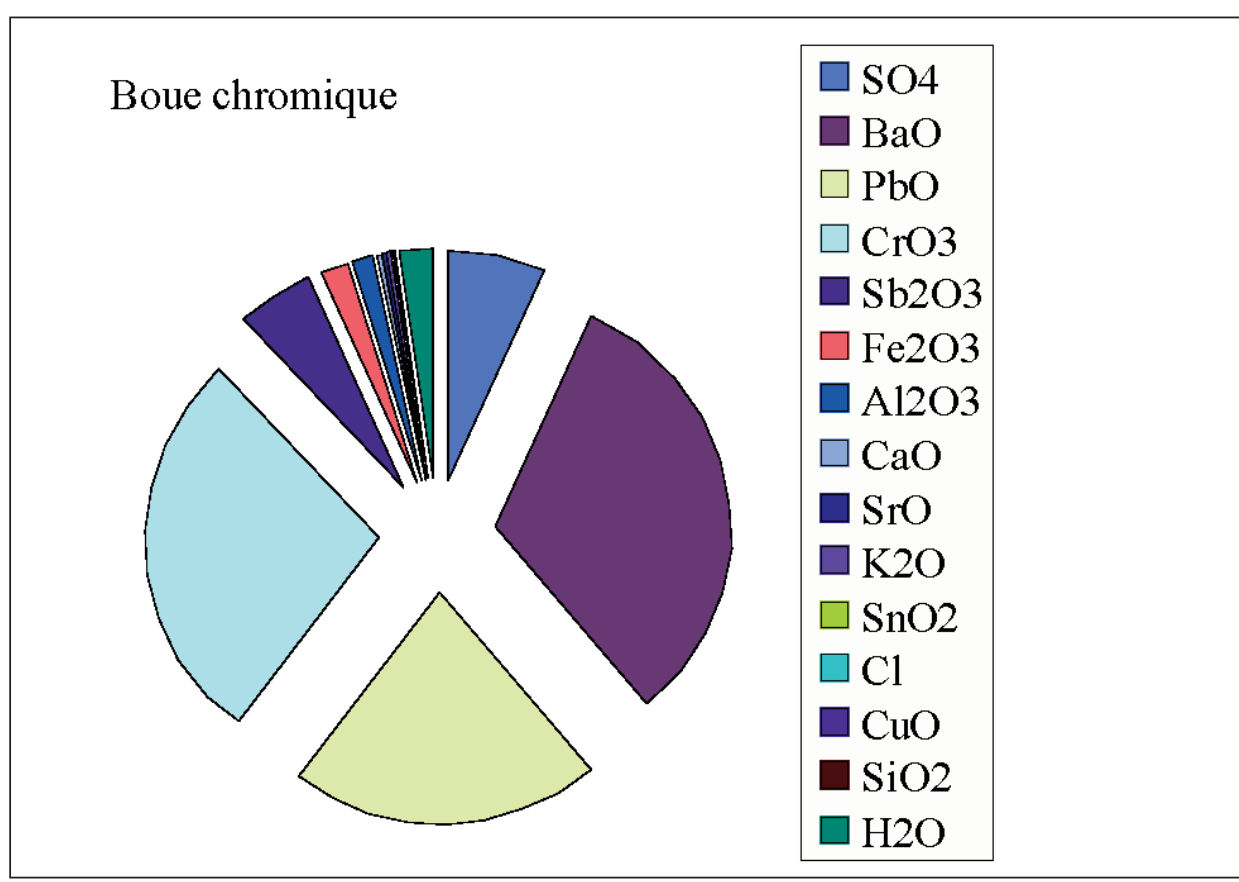

Contaminants de la matière recyclée

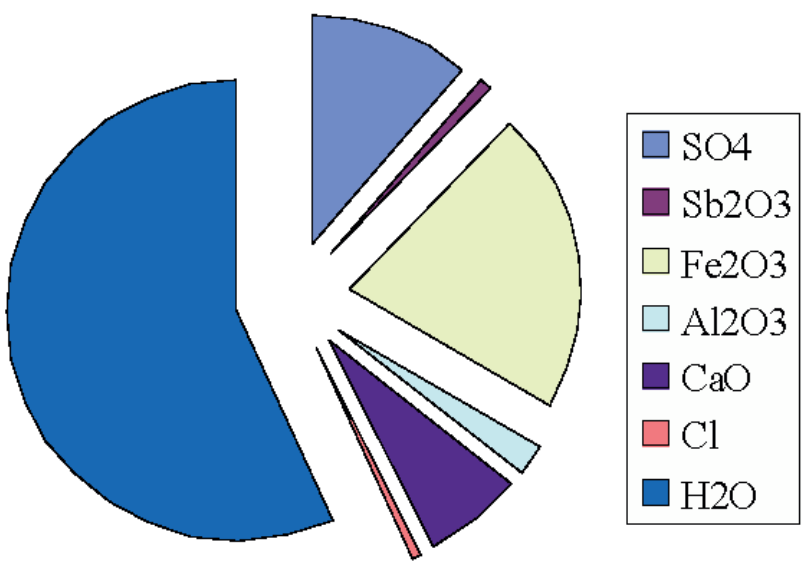

\section{Etape 1: Oxydation - séchage}

Le chrome (III) précipité se trouve sous forme de polyanions $\left(\mathrm{Cr}_{\mathrm{n}}(\mathrm{OH})_{3 \mathrm{n}+\mathrm{m}}\right)^{\mathrm{m}-}$ très hydratés, la première étape sera une dissolution en $\mathrm{H}_{2} \mathrm{SO}_{4}$ pour permettre l'oxydation électrochimique sur anode $(\mathrm{Sn}-\mathrm{Pb}) \mathrm{O}_{2-\mathrm{x}}$. On ajoute un minimum d'acide concentré au solide contenant encore une large quantité d'eau, l'oxydation anodique transforme le chrome trivalent en chromate, dichromate ou acide chromique selon les constituants du solide initial. Ce premier produit est séché.

\section{Etape 2: Substitution - évaporation}

Le chrome (VI) obtenu précédemment peut s'écrire $\mathrm{CrO}_{3} \cdot \mathrm{xH}_{2} \mathrm{O}$ ou $\mathrm{Cr}_{2} \mathrm{O}_{6} \cdot \mathrm{xH}_{2} \mathrm{O}$.
Cette étape consiste à substituer l'eau par le solvant d'extraction, soit l'obtention de composés de type $\mathrm{CrO}_{3} . \mathrm{yS}$ ou $\mathrm{Cr}_{2} \mathrm{O}_{6} . \mathrm{yS}$ par élimination concourante de l'eau présente par évaporation.

\section{Etape 3: Filtration - lavage - évaporation}

Le mélange liquide-solide obtenu est filtré, le solide est rincé par une fraction de solvant pur et recueilli pour une étape subséquente (5), le produit attendu est ainsi concentré dans la phase organique sous forme dissoute. Le solvant est éliminé par évaporation, le produit solide de ce séchage est recueilli pour une recristallisation en milieu aqueux.
Fig. 1. Composition de boue chromique avant recyclage

Fig. 2. Matière recyclée: $13 \%$ de contaminants et $87 \%$ de $\mathrm{CrO}_{3}$.

\section{Etape 4: Recristallisation}

Le solide précédent est dissout dans l'eau et recristallisé.

\section{Etape 5: Extraction et réduction des chromates insolubles}

Les chromates résiduels encore contenus dans le filtre utilisé à l'étape 3 sous forme de sels de plomb et de baryum, sont extraits par déplacement d'équilibre pour former le dichromate soluble, qui subit une réduction électrochimique, par exemple en utilisant le compartiment cathodique de l'électrodialyseur utilisé pour l'oxydation décrite à la première étape. Les figures 3 et 4 illustrent respectivement les produits initiaux et finaux du recyclage.

\section{Evaluation énergétique}

Le coût du procédé de recyclage, notamment du point de vue énergétique, sera particulièrement dépendant de la quantité de chrome trivalent à oxyder ainsi que de la teneur en eau, et bien sûr de la teneur en chrome total. A titre d'exemple, un solide contenant $1 \mathrm{kM}$ de 
chrome total par tonne (5.2\% masse), $6 \mathrm{kM}$ d'eau par $\mathrm{kM}$ de chrome trivalent et $1 \mathrm{kM}$ d'eau par kM de chrome hexavalent, pour lequel le schéma précédent est appliqué amène au bilan estimatif suivant:

Composition initiale
a) $100 \%$
Cr(III)
b) $50 \%$
Cr(III)
c) $100 \%$

\section{étape 1 \\ $1.30 \mathrm{GJ} / \mathrm{T}$ \\ $0.65 \mathrm{GJ} / \mathrm{T}$}

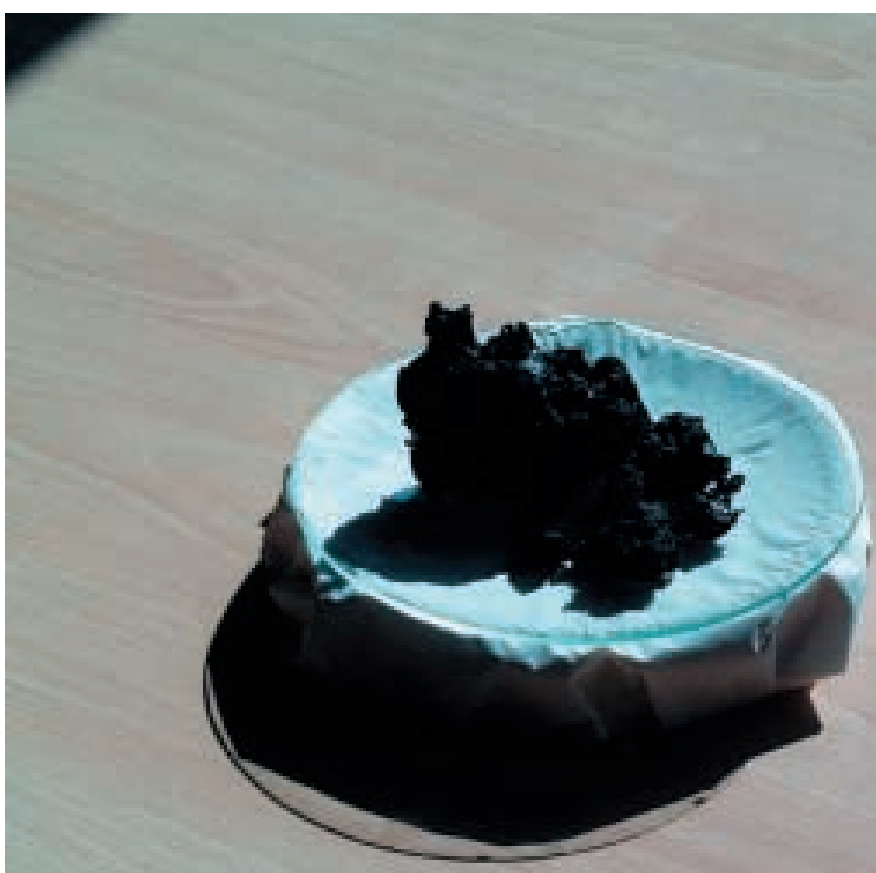

Fig. 3. Illustration d'un lot de boue chromique avant recyclage
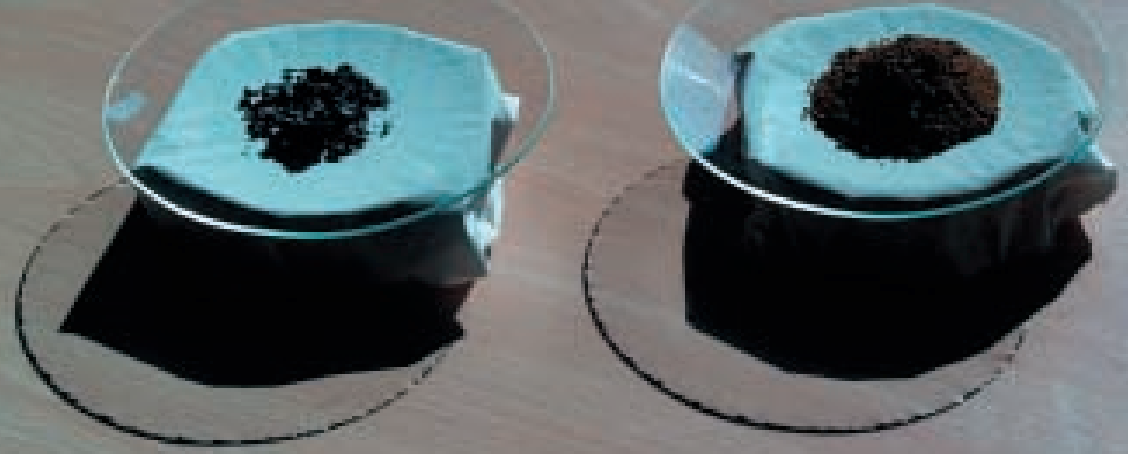

ramenés à la tonne de $\mathrm{CrO}_{3}$ recyclé, nous obtenons respectivement $35.8 \mathrm{GJ} / \mathrm{T}, 21.7$ GJ/T, 11.4 GJ/T. Ces valeurs tendent vers environ 10.6 GJ/T, pour des teneurs plus élevées en chrome hexavalent (> $3 \mathrm{kM}$ $\mathrm{Cr}(\mathrm{VI}) / \mathrm{T})$.

\section{Discussion}

Il faut prendre avec prudence les valeurs estimées jusqu'ici, cependant on confirme la valeur intrinsèque des boues chromiques lorsque l'on compare les coûts respectivement du recyclage et de l'extraction minière. Ainsi, une tonne d'oxyde de chrome hexavalent recyclé permet d'économiser de 25 à $60 \%$ de l'énergie consommée pour la production à partir du minerai. Le projet n'est que dans sa première phase de réalisation, nous allons nous attacher notamment à l'optimisation de l'extraction, et à l'évaluation d'alternatives technologiques de recyclage des solvants pour en abaisser les coûts d'exploitation.

L'application de ce procédé au recyclage des effluents concentrés ne sera économiquement viable, que si on trouve sur leur lieu d'utilisation des moyens de les concentrer jusqu'au solide, par exemple en utilisant les volumes d'air ventilés. La création d'un site d'exploitation industrielle est soumise aux caractéristiques du marché, soit les volumes à traiter et particulièrement la distribution statistique des teneurs en chrome hexavalent. Si on peut estimer les volumes à travers les données statistiques des flux de déchets, bien que les volumes qui sont traités par réduction chimique n'y apparaissent pas, les teneurs y relatives sont hors de portée car malheureusement les statistiques actuelles ne sont pas pondérées par les concentrations, alors que dans la pratique chaque lot entrant à fin de traitement physico-chimique, ou d'exportation pour stockage en dépôts agréés, est caractérisé par analyse.

Il n'en demeure pas moins que ces déchets sont parfois tout aussi concentrés que les meilleurs gisements miniers, et qu'il paraît absurde de consommer à la fois des réactifs chimiques et de l'énergie pour les rendre inertes pour les stocker dans les dépôts agréés pour matières stabilisées.

Fig. 4. Illustration de la matière recyclée et des déchets ultimes produits lors du recyclage. 